\title{
Recognition of Human Actions from RGB-D Videos Using a Reject Option
}

\author{
Vincenzo Carletti, Pasquale Foggia, Gennaro Percannella, \\ Alessia Saggese, and Mario Vento \\ Dept. of Information Eng., Electrical Eng. and Applied Mathematics (DIEM), \\ University of Salerno, Italy \\ \{vcarletti,pfoggia, pergen, asaggese, mvento\}@unisa.it
}

\begin{abstract}
In this paper we propose a method for recognizing human actions by using depth images acquired through a Kinect sensor. The depth images are represented through the combination of three sets of well-known features, respectively based on $\mathrm{Hu}$ moments, depth variations and the $\mathfrak{R}$ transform, an enhanced version of the Radon transform. A GMM classifier is adopted and finally a reject option is introduced in order to improve the overall reliability of the system. The proposed approach has been tested over two datasets, the Mivia and the MHAD, showing very promising results.
\end{abstract}

\section{Introduction}

The need for security in different applications fields, ranging from surveillance to patient monitoring systems, has led to a growing interest in those applications able to automatically interpret human behaviors. In this field, human action recognition is among the most challenging tasks.

According to [11], an action can be represented following two different approaches: local representation and global representation. Local representation is based on a bottom-up approach: the spatio-temporal interest points are extracted from the entire scene and local descriptors are then computed and combined into a final representation. For instance, a Microsoft Kinect sensor is used in [18] and [14]: the former proposed a novel 4D local spatio-temporal feature which combines both intensity and depth information; in the latter the features are based on human pose and motion, as well as on image and point-cloud information. Furthermore, the authors create a two-layer Maximum Entropy Markov strategy for modeling an activity as a set of sub-activities and exploit a dynamic programming approach for the inference. In [6] an unsupervised feature learning is proposed: the spatio-temporal features are learnt directly from unlabeled video data by means of an extension of the Independent Subspace Analysis algorithm. The main success of all these methods based on local features is that any kind of preprocessing methods, such as motion segmentation and tracking, can be avoided. Furthermore, such methods are invariant to changes in viewpoint, person appearance and partial occlusions. The main lack, however, lies in their computational burden: these techniques are usually very slow, and their applicability to real-time systems is not really feasible. For instance, in [6] the computation needs 0.44 seconds for each frame, 0.10 seconds only if the implementation is optimized for a GPU.

A. Petrosino, L. Maddalena, P. Pala (Eds.): ICIAP 2013 Workshops, LNCS 8158, pp. 436 445, 2013.

(C) Springer-Verlag Berlin Heidelberg 2013 
On the contrary, in global representation based approaches the visual observation focuses on a Region of Interest (ROI): the person is located in the scene by applying background subtraction or tracking techniques; the descriptors in this case are usually derived from silhouettes, edges or optical flow. For instance, in [2] and [17] a Radon transform and an extended version, the $\mathfrak{R}$ transform, have been respectively adopted. In particular, the $\mathfrak{R}$ transform is invariant to scale and translation and, as experimentally demonstrated in [17], outperforms methods using silhouette-based moment descriptors (like, for instance, Invariant Moments, Zernike Moments, Pseudo-Zernike Moments and Wavelet Moments) without increasing the computational cost. In [9] each action is represented by its 3D volume (two spatial and one temporal dimensions); the characterization of this volume is performed by geometrical moments based on Hu moments [1]. In [8] RGB-D images are considered. Such approaches significantly improve the extraction of the silhouette, being insensitive to the typical problems of the detection phase (like, for instance, illuminance conditions changes or camouflage). However, the main problem in the approaches based on global representations lies in the fact that such techniques are sensible to noise, partial occlusions and variations in viewpoint. In order to overcome these limitations, global representations are often enriched by a grid-based approach: the ROI is partitioned into cells in order to also obtain local information about the part of the objects in the scene.

The proposed method belongs to the last category: RGB-D images acquired by a Kinect are processed in order to extract the moving object silhouette. A first original aspect of the method is the use of a combination of three well-known different sets of features, respectively based on the $\mathfrak{R}$ transform, depth variations and $\mathrm{Hu}$ moments. The first set is good at capturing properties related to the alignment of subregions of the image, while the second set describes in a more global way the overall distribution of the pixels; thus they are quite complementary in their nature, and their combination improves the discriminant ability with respect to each set taken separately. Also note that both sets are based on integral criteria, and so are quite robust with respect to noise.

The classification is performed by means of a Gaussian Mixture Model (GMM) classifier. A second major novelty of the method is the addition, after the classifier, of a module that estimates the reliability of the single classification response. This module is able to reject (i.e. refrain from recognizing an action) when observing a sample whose reliability is low. In this way the system avoids issuing erroneus action reports for ambiguous or unstable situations, such as during the transition between two different actions, thus improving the classification performance.

\section{The Proposed Method}

This section will detail the proposed method: two main phases can be identified, as shown in Figure 1; a low-level phase (colored in orange in the figure), aimed at extracting features starting from the analysis of raw data, and a high-level phase (in blue in the figure), devoted to identify the actions of the object of interest, by analyzing the extracted features. More details about each module will be provided in the following. 


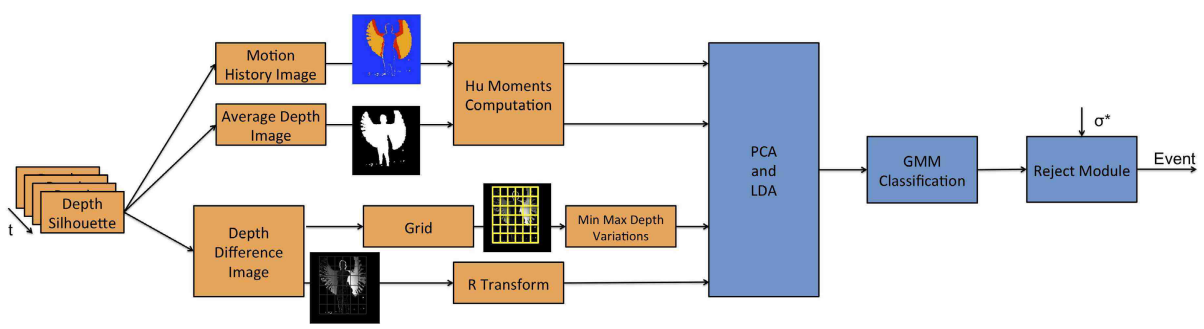

Fig. 1. Overview of the proposed method

\subsection{Low-Level Phase}

This phase includes acquisition of the images, their representation, and feature extraction. Each of these steps is described in the following.

Acquisition: The raw images are acquired using a Kinect, an affordable (under \$150) input device produced by Microsoft, that provides both a RGB image and a depth image obtained by means of an infrared image sensor combined with a structured infrared light source.

Representation: The depth image $D$ is processed to obtain three different representations, the Motion History Image (MHI), the Average Depth Image (ADI) and the Depth Difference Image (DDI); these three representations will be used as the basis for feature extraction. The Motion History Image MHI [4] is used to capture into a single and static image the sequence of motions. The value of MHI at time $t$ and position $(x, y)$ is updated as follows:

$$
\operatorname{MHI}(x, y, t)=255
$$

if point $(x, y)$ passed from background to foreground at time $t$, and

$$
M H I(x, y, t)=\max \{M(x, y, t-1)-\tau, 0\}
$$

otherwise; $\tau$ is a constant.

Given an observation window of $N$ temporally adjacent images, the Average Depth Image $A D I(x, y, t)[8]$ is the average depth at position $(x, y)$ over the images at times $t-N+1, \ldots, t$.

Finally, the Depth Difference Image DDI [8] is used to evaluate the motions changes in the depth dimension:

$$
D D I(x, y, t)=D_{\max }(x, y, t)-D_{\min }(x, y, t)
$$

where $D_{\max }(x, y, t)$ and $D_{\min }(x, y, t)$ are respectively the maximum and minimum depth for position $(x, y)$ over the images at times $t-N+1, \ldots, t$.

It is worth pointing out that $N$ in our experiments is set to one second, corresponding to 25 frames. Furthermore, in [8] it has been experimental evaluated that the optimal value of $\tau$ is $(256 / N)-1$. 
Features Extraction: Once obtained the derived images, three different kinds of features have been extracted. In particular, Hu moments [1] have been chosen to represent the MHI and ADI, while the DDI is represented through two different kinds of features: the $\mathfrak{R}$ transform [15] and the Min-Max Depth Variations [8]. The former is an extended Radon transform; its main advantages lie in its low computational complexity and its geometric invariance: in particular, such a descriptor is robust with respect to the errors of the detection phase, such as disjoint silhouettes and holes in the shape. The latter is obtained by hierarchical partitioning the box containing the silhouette into cells of equal size and by computing the maximum and the minimum values in each cell (see Figure 2).

It is worth pointing out that the features extraction step results in a vector composed by 303 features: 14 for the $\mathrm{Hu}$ moments, 181 representing the $\mathfrak{R}$ transform and 108 encoding the Min-Max Depth Variations over the grid.

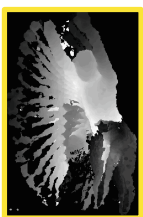

(a)

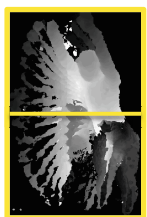

(b)

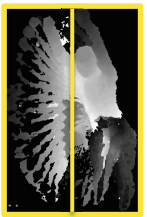

(c)

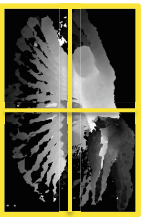

(d)

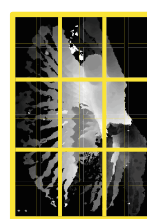

(e)

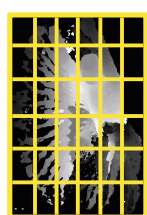

(f)

Fig. 2. Min Max Depth Variations Features extracted with hierarchical grids

\subsection{High-Level Phase}

The high-level phase is composed by feature reduction, classification and reject module, described below.

Feature reduction: In order to improve the performance of the system, both in terms of overall accuracy and computational cost, a Principal Component Analysis (PCA) and a Linear Discriminant Analysis (LDA) are used for reducing the dimensionality of the feature vectors. This combination has been often used for face recognition applications (the reader can refer, for instance, to [7]), showing a significant improvement in the overall accuracy of the methods.

Classification: Once obtained the reduced features vector, a Gaussian Mixture Model (GMM) classifier is used for the final step. During the training step, the distribution of each class is estimated by maximizing the likelihood. During the classification phase, the likelihood for each class is computed; before assigning the observation to the class for which the likelihood is maximum, the system computes the estimation of the reliability of the obtained results $\psi$. If $\psi$ is below a given threshold $\sigma^{*}$, then the sample will be rejected. According to the method proposed in [5], $\psi$ can be computed as the combination of two different terms, $\psi_{a}$ and $\psi_{b}$, that account for the typical situations that usually make low the reliability of a classifier: (a) the sample significantly differs from those present in the training set; (b) the point representing the sample in 
the features space lies at the boundary between two classes. In particular, for the GMM classifier $\psi_{a}$ can be computed as

$$
\psi_{a}=O_{w i n}
$$

being $O_{\text {win }}$ the maximum likelihood computed. Similarly, $\psi_{b}$ can be computed as

$$
\psi_{b}=O_{\text {win }}-O_{2 w i n}
$$

where $\mathrm{O}_{2 \text { win }}$ is the likelihood of the second best class. In conclusion, for the GMM classifier,

$$
\psi=\min \left\{O_{w i n}, O_{w i n}-O_{2 w i n}\right\}=\psi_{b} .
$$

Reject Module: This step compares the reliability indicator $\psi$ with a threshold $\sigma$, and rejects the sample if it is below the threshold. The reject option has been successfully used in other application fields, ranging from audio-surveillance [3] to biomedical analysis [13]. At our knowledge, it is used for the first time in this paper for actions recognition purposes. The method for choosing an optimal threshold $\sigma^{*}$ is described in [5]. The main idea is that $\sigma^{*}$ can be computed by maximizing a function $P$, which measures the classification effectiveness in the considered application domain, in terms of recognition rate $R_{c}$, error rate $R_{e}$ and reject rate $R_{r}$. Assuming a linear dependence for $P$ on $R_{c}, R_{e}$ and $R_{r}, P$ can be computed as follows:

$$
P=C_{c} \cdot\left(R_{c}-R_{c}^{0}\right)-C_{e} \cdot\left(R_{e}-R_{e}^{0}\right)-C_{r} \cdot R_{r},
$$

being $R_{c}^{0}$ and $R_{e}^{0}$ respectively the recognition rate and the error rate at 0 -reject. Furthermore, $C_{e}, C_{r}$ and $C_{c}$ respectively represent the cost of each error, of each rejection and the gain of each correct classification. Of course, such costs strongly depend on the particular application domain.

It should be clear that $R_{c}, R_{e}$ and $R_{r}$ depend on $\sigma$. It means that also $P$ is a function of the reject threshold. Since this function is not available in analytic form, the optimal threshold $\sigma^{*}$ has to be experimentally evaluated by means an exhaustive search among the tabulated values of $P(\sigma)$.

\section{Experimental Results}

In the last years, a lot of datasets for action recognition have been proposed. However, they usually have two limitations with respect to the needs of our experimentation: first, the RGB-D images are not available [12] or, if available, the background is not included in the dataset [16]. This issue is justified by the fact that a lot of methods are not based on background subtraction strategies, since they directly extract the objects skeletons from the images. The second limitation depends on the typology of the extracted data: as a matter of fact, we are mainly interested in actions with a high semantic level (eating, drinking etc.). Most of the available datasets, instead, mainly focus on actions with low semantic level, like running, sitting down, standing up and so on. For the above mentioned reasons, we built our own dataset, from now on denoted as the Mivia Dataset; 
however we also used an existing dataset, for making our results easier to compare with other. Thus, the used datasets were:

Mivia Dataset: it has been acquired in the Mivia lab and it is available at the following link: http://mivia.unisa.it/datasets/video-analysis-datasets/mivia-action-dataset/. It is composed by 7 high-level actions performed by 14 subjects ( 7 males and 7 females). All the subjects performed 5 repetitions of each action, so resulting in about 500 repetitions; the approximated length of each action is reported in Table 1

Berkeley Multimodal Human Action Detection (MHAD) [10]: it contains 11 lowlevel actions performed by 7 male and 5 female subjects. All the subjects performed 5 repetitions of each action, yielding about 660 action sequences which correspond to about 82 minutes of total recording time.

Table 1 summarizes the actions recorded in each dataset, while Figure 3 shows some examples.

Table 1. Description of the Berkeley MHAD (a) and of the Mivia (b) Datasets

\begin{tabular}{|c|c|c|}
\hline ID & Action & $\begin{array}{c}\text { Length per } \\
\text { Recording }\end{array}$ \\
\hline A1 & Bending - hands up all the way down & 12 secs \\
\hline A2 & Clapping hands & 5 secs \\
\hline A3 & Jumping in place & 5 secs \\
\hline A4 & Jumping jacks & 7 secs \\
\hline A5 & Punching (boxing) & 10 secs \\
\hline A6 & Sit down & 2 secs \\
\hline A7 & Sit down then stand up & 15 secs \\
\hline A8 & Stand up & 2 secs \\
\hline A9 & Throwing a ball & 3 secs \\
\hline A10 & Waving - one hand (right) & 7 secs \\
\hline A11 & Waving - two hands & 7 secs \\
\hline
\end{tabular}

(a)

\begin{tabular}{|c|c|c|}
\hline ID & Action & $\begin{array}{c}\text { Length per } \\
\text { Recording }\end{array}$ \\
\hline B1 & Opening a jar & 2 sec \\
\hline B2 & Drinking & 3 secs \\
\hline B3 & Sleeping & 3 secs \\
\hline B4 & Random Movements & 11 secs \\
\hline B5 & Stopping & 7 secs \\
\hline B6 & Interacting with a table & 3 secs \\
\hline B7 & Sitting & 3 secs \\
\hline
\end{tabular}

(b)

For each dataset, two different experimentations has been conducted: in the first case, we did include the person to be tested in the training set; namely, one of the repetitions of each action performed by the person being tested was included in the training set, together with all the repetitions by all the other persons; the test has been repeated for all the persons in the database, and the average performance has been reported. This case represents the situation in which the person whose actions have to be recognized is known to the system, a situation that occurs in many applications (e.g. home monitoring of an elderly person). In the second case, the person to be tested is not included in the training set, which is formed by the actions of all the other persons. This case models the situation in which the actions of unknown persons have to be recognized, that occurs in applications like surveillance of public places.

The results, in terms of true positive rate, for the different sets of features (in particular $\mathfrak{R}$ features, used in [2], depth-based features (Hu Moments and Min-Max Depth Variation), used in [8], and their combination, first introduced in this paper) are shown in Tables 2 for the Mivia (a)(b) and the MHAD dataset (c)(d) respectively. 


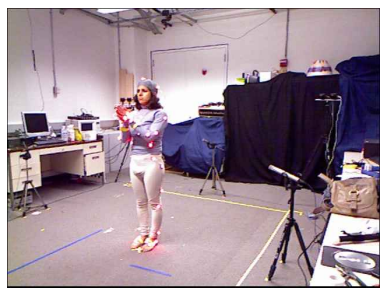

(a)

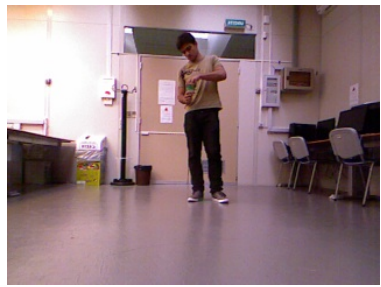

(d)

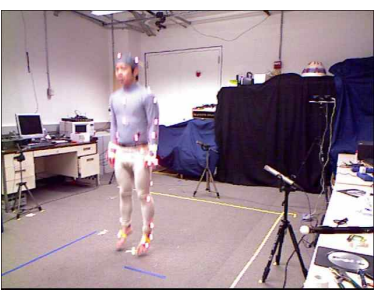

(b)

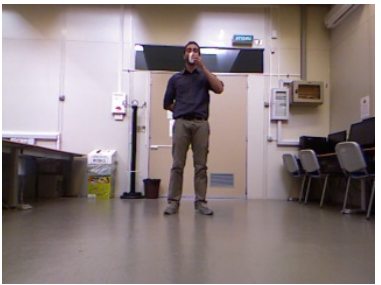

(e)

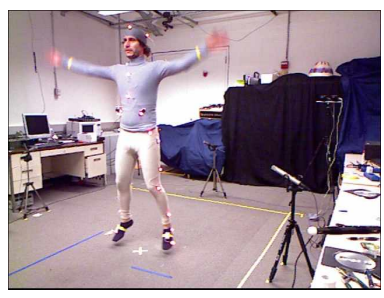

(c)

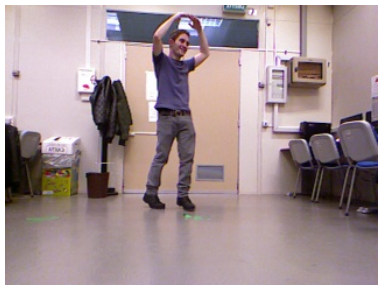

(f)

Fig. 3. Some examples of the Berkeley MHAD Dataset (A2 (a), A3 (b), A4 (c)) and of the Local Dataset (B1 (e), B2 (f), B4 (g))

On the Mivia dataset, the absolute performance for all the methods is quite high for the case of the test person included in the training set and (as expected) slightly worse for the case of the test person excluded. On the other hand, the performance on MHAD is lower; it is worth pointing out that the MHAD is a very challenging dataset: actions like, for instance, Sit down and Sit down then stand up are very similar to each other (the first two seconds are exactly the same). However, the interesting point is that in both the test cases, and for both the datasets, the proposed method results in a measurable improvement of the classification accuracy; the improvement is usually between $2 \%$ and $3 \%$, but for some actions it is quite larger (e.g. for action A5 of the MHAD database, in the unknown person scenario, the accuracy improvement is $18 \%$ ).

Finally, we evaluated the impact of the reject option on the classifier performance, by comparing the error rates of a zero-reject classifier with the error and reject rates of a classifier using the optimal reject threshold previously defined (for this experiment we set the costs as $C_{r}=2, C_{e}=5$ and $C_{c}=1$ ).

Table 3 shows the obtained results. As it can be seen, for the Mivia Dataset the improvements were negligible. However, on the more difficult MHAD Dataset the reject option is able on the average to reduce the error rate by an additional $2.3 \%$, thus confirming its usefulness.

Finally, as for the computational cost, we verified that the proposed method is able to run at $10 \mathrm{fps}$ on a MacBook Pro equipped with Intel Core 2 Duo running at $2.4 \mathrm{GHz}$, so confirming its applicability in real frameworks. 
Table 2. Recognition rates over the Mivia dataset (a)(b) and over the Berkeley MHAD dataset (c)(d) with a different feature set ( $\Re$ Features, Depth Features and the combination of both), with and without the person under test in the training set

\begin{tabular}{|c|c|c|c|}
\hline \multicolumn{4}{|c|}{ The test person is in the training set } \\
\hline & $\Re$ Features & Depth Features & $\begin{array}{c}\text { Proposed } \\
\text { Method }\end{array}$ \\
\hline B1 & 47.453 & 91.491 & $\mathbf{9 3 . 3 4 0}$ \\
\hline B2 & 39.068 & 93.825 & $\mathbf{9 4 . 6 6 0}$ \\
\hline B3 & 97.913 & 99.986 & $\mathbf{9 9 . 9 8 7}$ \\
\hline B4 & 85.858 & $\mathbf{9 8 . 8 0 9}$ & 98.709 \\
\hline B5 & 89.225 & 98.791 & $\mathbf{9 9 . 2 2 2}$ \\
\hline B6 & 35.400 & 98.475 & $\mathbf{9 8 . 9 5 0}$ \\
\hline B7 & 65.596 & 98.756 & $\mathbf{9 8 . 8 0 8}$ \\
\hline
\end{tabular}

(a)

\begin{tabular}{|c|c|c|c|}
\hline \multicolumn{4}{|c|}{ The test person is in the training set } \\
\hline & $\Re$ Features & Depth Features & $\begin{array}{c}\text { Proposed } \\
\text { Method }\end{array}$ \\
\hline A1 & 56.462 & 83.829 & $\mathbf{8 6 . 4 8 3}$ \\
\hline A2 & 82.999 & 94.405 & $\mathbf{9 5 . 1 9 5}$ \\
\hline A3 & 43.124 & 72.556 & $\mathbf{8 3 . 2 2 3}$ \\
\hline A4 & 84.020 & 88.273 & $\mathbf{9 1 . 5 1 8}$ \\
\hline A5 & 46.405 & 87.504 & $\mathbf{8 8 . 9 9 9}$ \\
\hline A6 & 24.020 & 66.612 & $\mathbf{7 5 . 4 5 6}$ \\
\hline A7 & 34.110 & $\mathbf{8 1 . 8 4 3}$ & 80.327 \\
\hline A8 & 38.912 & $\mathbf{8 4 . 8 5 4}$ & 81.829 \\
\hline A9 & 24.985 & 77.894 & $\mathbf{8 1 . 0 3 8}$ \\
\hline A10 & 80.270 & 95.823 & $\mathbf{9 6 . 7 2 6}$ \\
\hline A11 & 71.628 & 83.208 & $\mathbf{8 9 . 2 3 3}$ \\
\hline
\end{tabular}

(c)

\begin{tabular}{|c|c|c|c|}
\hline \multicolumn{4}{|c|}{ The test person is NOT in the training set } \\
\hline & $\mathfrak{R}$ Features & Depth Features & $\begin{array}{c}\text { Proposed } \\
\text { Method }\end{array}$ \\
\hline B1 & 33.574 & 82.604 & $\mathbf{8 3 . 6 7 7}$ \\
\hline B2 & 23.356 & $\mathbf{7 7 . 7 9 4}$ & 73.967 \\
\hline B3 & 80.297 & 97.694 & $\mathbf{9 9 . 8 9 3}$ \\
\hline B4 & 85.234 & $\mathbf{9 8 . 0 2 3}$ & 97.526 \\
\hline B5 & 85.532 & $\mathbf{9 1 . 5 3 3}$ & 88.287 \\
\hline B6 & 23.442 & 96.360 & $\mathbf{9 8 . 0 3 3}$ \\
\hline B7 & 43.529 & 93.090 & $\mathbf{9 4 . 7 5 3}$ \\
\hline
\end{tabular}

(b)

\begin{tabular}{|c|c|c|c|}
\hline \multicolumn{4}{|c|}{ The test person is NOT in the training set } \\
\hline & $\mathfrak{R}$ Features & Depth Features & $\begin{array}{c}\text { Proposed } \\
\text { Method }\end{array}$ \\
\hline A1 & 49.282 & 69.942 & $\mathbf{7 4 . 9 7 4}$ \\
\hline A2 & 44.101 & 54.580 & $\mathbf{5 8 . 9 6 9}$ \\
\hline A3 & 22.971 & 41.651 & $\mathbf{4 7 . 4 5 0}$ \\
\hline A4 & $\mathbf{7 7 . 8 1 4}$ & 69.151 & 67.886 \\
\hline A5 & 33.051 & 52.447 & $\mathbf{7 0 . 2 0 1}$ \\
\hline A6 & 12.540 & 32.554 & $\mathbf{3 5 . 4 6 8}$ \\
\hline A7 & 21.803 & $\mathbf{6 6 . 0 8 6}$ & 59.943 \\
\hline A8 & 19.130 & 47.467 & $\mathbf{4 8 . 9 5 7}$ \\
\hline A9 & 6.407 & $\mathbf{5 8 . 1 6 7}$ & 57.384 \\
\hline A10 & 44.783 & 78.271 & $\mathbf{7 9 . 5 1 2}$ \\
\hline A11 & 56.996 & 62.380 & $\mathbf{6 4 . 9 0 3}$ \\
\hline
\end{tabular}

(d)

Table 3. Performance of the system over the Mivia and the MHAD datasets, in terms of Error Rate (with and without reject) and Reject Rate

\begin{tabular}{|c|c|c|c|}
\hline \multicolumn{4}{|c|}{ Mivia Dataset } \\
\hline Class & $\begin{array}{c}\text { Error Rate } \\
\text { without reject }\end{array}$ & $\begin{array}{c}\text { Error Rate } \\
\text { with reject }\end{array}$ & Reject rate \\
\hline B1 & 6.66 & 5.53 & 2.49 \\
\hline B2 & 5.34 & 4.35 & 1.83 \\
\hline B3 & 0.01 & 0.01 & 0.01 \\
\hline B4 & 1.29 & 1.20 & 0.14 \\
\hline B5 & 0.78 & 0.52 & 0.63 \\
\hline B6 & 1.05 & 0.89 & 0.17 \\
\hline B7 & 1.19 & 1.16 & 0.04 \\
\hline Total & 2.33 & 1.95 & 0.76 \\
\hline
\end{tabular}

\begin{tabular}{|c|c|c|c|}
\hline \multicolumn{4}{|c|}{ MHAD Dataset } \\
\hline Class & $\begin{array}{c}\text { Error Rate } \\
\text { without reject }\end{array}$ & $\begin{array}{c}\text { Error Rate } \\
\text { with reject }\end{array}$ & Reject rate \\
\hline A1 & 13.52 & 11.39 & 2.99 \\
\hline A2 & 4.80 & 3.66 & 1.40 \\
\hline A3 & 16.78 & 13.22 & 5.32 \\
\hline A4 & 8.48 & 7.36 & 1.64 \\
\hline A5 & 11.00 & 9.99 & 1.47 \\
\hline A6 & 24.54 & 21.94 & 5.06 \\
\hline A7 & 19.67 & 17.82 & 3.41 \\
\hline A8 & 18.17 & 15.11 & 4.84 \\
\hline A9 & 18.96 & 16.22 & 4.35 \\
\hline A10 & 3.27 & 2.58 & 1.16 \\
\hline A11 & 10.77 & 8.56 & 3.01 \\
\hline Total & 15.25 & 12.90 & 3.59 \\
\hline
\end{tabular}




\section{Conclusions}

In this paper we proposed a framework for recognizing human activities by analyzing depth images extracted from a Kinect sensor. The experimental results show that the combination of the three sets of features, respectively based on Hu moments, depth variations and $\mathfrak{R}$ transform, consistently improved the performance of the system. The overall reliability is further enhanced by introducing a reject option, which has been exploited for the first time in this paper in the field of action recognition.

Acknowledgment. This research has been partially supported by A.I.Tech s.r.l. (a spinoff company of the University of Salerno, www . aitech-solutions.eu).

\section{References}

1. Bobick, A.F., Davis, J.W.: The recognition of human movement using temporal templates. IEEE Trans. Pattern Anal. Mach. Intell. 23(3), 257-267 (2001), http://dx.doi.org/10.1109/34.910878

2. Chen, Y., Wu, Q., He, X.: Human action recognition based on radon transform. In: Lin, W., Tao, D., Kacprzyk, J., Li, Z., Izquierdo, E., Wang, H. (eds.) Multimedia Analysis, Processing and Communications. SCI, vol. 346, pp. 369-389. Springer, Heidelberg (2011)

3. Conte, D., Foggia, P., Percannella, G., Saggese, A., Vento, M.: An ensemble of rejecting classifiers for anomaly detection of audio events. In: 2012 IEEE Ninth International Conference on Advanced Video and Signal-Based Surveillance (AVSS), pp. 76-81 (September 2012)

4. Davis, J.: Hierarchical motion history images for recognizing human motion. In: Proceedings of the IEEE Workshop on Detection and Recognition of Events in Video, pp. 39-46 (2001)

5. Foggia, P., Sansone, C., Tortorella, F., Vento, M.: Multiclassification: reject criteria for the bayesian combiner. Pattern Recognition 32(8), 1435-1447 (1999), http://www.sciencedirect.com/science/article/pii/ S0031320398001691

6. Le, Q., Zou, W., Yeung, S., Ng, A.: Learning hierarchical invariant spatio-temporal features for action recognition with independent subspace analysis. In: 2011 IEEE Conference on Computer Vision and Pattern Recognition (CVPR), pp. 3361-3368 (June 2011)

7. Li, J., Zhao, B., Zhang, H.: Face recognition based on pca and lda combination feature extraction. In: 2009 1st International Conference on Information Science and Engineering (ICISE), pp. 1240-1243 (2009)

8. Megavannan, V., Agarwal, B., Babu, R.: Human action recognition using depth maps. In: 2012 International Conference on Signal Processing and Communications (SPCOM), pp. 1-5 (July 2012)

9. Mokhber, A., Achard, C., Qu, X., Milgram, M.: Action recognition with global features. In: Sebe, N., Lew, M., Huang, T.S. (eds.) HCI/ICCV 2005. LNCS, vol. 3766, pp. 110-119. Springer, Heidelberg (2005)

10. Ofli, F., Chaudhry, R., Kurillo, G., Vidal, R., Bajcsy, R.: Berkeley mhad: A comprehensive multimodal human action database. In: IEEE Workshop on Applications on Computer Vision (WACV). IEEE (2013)

11. Poppe, R.: A survey on vision-based human action recognition. Image Vision Comput. 28(6), 976-990 (2010), http: / / dx.doi.org/10.1016/j.imavis.2009.11.014

12. Schuldt, C., Laptev, I., Caputo, B.: Recognizing human actions: a local svm approach. In: Proceedings of the 17th International Conference on Pattern Recognition, ICPR 2004, vol. 3 , pp. 32-36 (August 2004) 
13. Soda, P., Iannello, G., Vento, M.: A multiple expert system for classifying fluorescent intensity in antinuclear autoantibodies analysis. Pattern Anal. Appl. 12(3), 215-226 (2009), http://dx.doi.org/10.1007/s10044-008-0116-z

14. Sung, J., Ponce, C., Selman, B., Saxena, A.: Unstructured human activity detection from rgbd images. In: ICRA, pp. 842-849. IEEE (2012)

15. Tabbone, S., Wendling, L., Salmon, J.P.: A new shape descriptor defined on the radon transform. Comput. Vis. Image Underst. 102(1), 42-51 (2006), http://dx.doi.org/10.1016/j.cviu.2005.06.005

16. Wang, J., Liu, Z., Wu, Y., Yuan, J.: Mining actionlet ensemble for action recognition with depth cameras. In: 2012 IEEE Conference on Computer Vision and Pattern Recognition (CVPR), pp. 1290-1297 (June 2012)

17. Wang, Y., Huang, K., Tan, T.: Human activity recognition based on $r$ transform. In: IEEE Conference on Computer Vision and Pattern Recognition, CVPR 2007, pp. 1-8 (June 2007)

18. Zhang, H., Parker, L.: 4-dimensional local spatio-temporal features for human activity recognition. In: 2011 IEEE/RSJ International Conference on Intelligent Robots and Systems (IROS), pp. 2044-2049 (September 2011) 